\title{
Previously Unreported Somatic Variants in Two Patients with Pleuropulmonary Blastoma with Metastatic Brain Recurrence
}

\author{
Michael Ferguson ${ }^{1}$, Jennifer Ivanovich ${ }^{1}$, Paige Stansell ${ }^{1}$, Terry Vik ${ }^{2}$, Amy Helvie ${ }^{3}$, Morgan \\ Schmitt $^{1}$, Kris Ann Schultz ${ }^{4}$, Louis Dehner ${ }^{5}$, Jamie Renbarger ${ }^{2}$, and Mark Marshall ${ }^{1}$ \\ ${ }^{1}$ Indiana University, School of Medicine \\ ${ }^{2}$ Indiana University School of Medicine \\ ${ }^{3}$ Indiana University Health \\ ${ }^{4}$ University of Minnesota \\ ${ }^{5}$ Washington University in Saint Louis
}

May 19, 2020

\begin{abstract}
Pleuropulmonary blastoma (PPB) is the most common primary lung tumor of childhood and is associated with somatic or germline DICER1 variants. Recurrent PPB, especially with brain metastases, are difficult to treat and survival is poor. Comprehensive genomic analyses of PPB have been limited in number and depth. The cases presented here identified additional oncogenic drivers from tumor sequencing that could be modulating tumor progression and response to therapy outside of known DICER1 mutations highlighting the need for upfront genomic analysis on all patients with PPB.
\end{abstract}

\section{Abstract}

Pleuropulmonary blastoma (PPB) is the most common primary lung tumor of childhood and is associated with somatic or germline DICER1 variants. Recurrent PPB, especially with brain metastases, are difficult to treat and survival is poor. Comprehensive genomic analyses of PPB have been limited in number and depth. The cases presented here identified additional oncogenic drivers from tumor sequencing that could be modulating tumor progression and response to therapy outside of known DICER1 mutations highlighting the need for upfront genomic analysis on all patients with PPB.

Introduction

Pleuropulmonary blastoma (PPB) is the most common primary lung tumor of childhood with $96 \%$ of tumors diagnosed prior to seven years of age and are associated with pathogenic somatic or germline DICER1 variants in most cases $[1,2]$. First described as an entity in 1988, its initial feature is a multi-locular cyst whose septa are composed of primitive mesenchymal cells which either progress to a primitive multi-patterned sarcoma with overgrowth of the cysts into a high grade neoplasm with anaplasia and p53 mutations or undergoes regression [3]. From this observation, familial predisposition, DICER1 germline mutation and the recognition of a family ofDICER1 -associated neoplasms emerged [3].

The current study documents other molecular aspects of PPB which are the emergence of distinct genomic findings in recurrent type III PPBs in two children.

Case Descriptions

Case 1 - A 2-year-old male initially presented with a persistent cough with subsequent computerized topography $(\mathrm{CT})$ scan revealing a $10 \times 9.8 \times 8.1 \mathrm{~cm}$ heterogeneous, hypodense mass occupying the left hemithorax. 
A biopsy revealed a high grade sarcoma with the primitive multi-pattern of PPB type III. Brain CT and bone scan were negative for metastatic disease at that time. The patient received 12 cycles of ifosfamide, vincristine, actinomycin-D and doxorubicin (IVADo) and underwent a left lower lobe resection at week 12. He subsequently received intra-cavitary cisplatin for local control. At 36 months post-treatment a $3 \mathrm{~cm}$ mass arising from the left ventricle was discovered by routine echocardiogram. The mass was resected and found to be recurrent (metastatic) PPB. He underwent re-induction chemotherapy with autologous stem cell rescue and was without recurrence for two years. Subsequently, he developed brain metastases, prompting a Precision Genomics consultation for tumor molecular analysis of these brain metastases. He then received irinotecan and pazopanib due to FGFR1 gene amplification and overexpression of TOPO1 protein. Genomic analysis was performed on a new brain metastasis and demonstrated an ETV6-NTRK fusion. He participated in a larotrectinib trial for 4 cycles before progression of disease and subsequent death nearly 7 years after initial diagnosis.

Case 2 - A 3-year-old female was admitted with a history of persistent cough and found to have a solid heterogeneous mass in the left chest measuring $10 \times 8.4 \times 10.5 \mathrm{~cm}$ on CT. A biopsy revealed a primitive sarcoma with rhabdomyoblastic features and anaplasia whose features were those of type III PPB. Brain MRI and bone scan failed to demonstrate metastatic disease, but an echocardiogram showed a multi-lobulated mass attached to the left atrium. She subsequently underwent resection of the cardiac mass. She then completed IVADo chemotherapy and received intra-cavitary cisplatin. Two months post-completion of intracavitary cisplatin, she was found to have multiple brain metastases for which she received gamma knife radiotherapy. She then received 2 cycles of ifosfamide, carboplatin, and etoposide post radiation. Utilizing metronomic chemotherapy, consisting of fenofibrate, thalidomide, alternating oral cyclophosphamide and etoposide, and every 2 week bevacizumab, she is continuing on therapy without recurrence [4].

Genetic Analysis and Results

Case 1. DNAseq, RNAseq and limited proteome analysis was performed on a recurrent brain tumor sample and whole-exome DNAseq and RNAseq was repeated from a second tumor sample from a subsequent recurrence (NantHealth) with matching germline sequencing. The first tumor sample showed amplification of FGFR1 (7x), TP53 p.R273H and high expression of TOP1 protein. From the sequencing of the second specimen, the presence of an additional ETV6-NRTK3 gene fusion was identified. Reanalysis of the DNA and RNA data from the first tumor sample revealed that the ETV6-NRTK3 fusion was present, but was not initially identified by NantHealth. A somatic AGO2 variant, AGO2 p.H443R, classified as unknown significance was identified in both tumor samples. DICER1 mutation was not found in the somatic and germline specimens.

Case 2. The tumor sample from the initial diagnosis was sequenced using the FoundationOne Heme panel. A number of known oncogenic alterations were identified in the sample: MDM2 amplification (30x), PIK3CA, p.Q546P, and PPP2R1A p.R183W. Germline analysis was performed at Ambry Genetics Laboratory and identified a pathogenic DICER1 variant, p.R676*.

Additional cancer relevant genomic findings are summarized in Table 1.

Discussion

DICER 1 germline pathogenic variants were discovered to harbor an increased risk for development of a variety of neoplasms, with PPB highlighted as the archetype [5-7]. Brain metastases, especially in recurrence, are difficult to treat and the survival rate in children remains low $[4,8]$, emphasizing the need to understand additional genomic drivers to develop novel treatments.

In the first case, the lack of either a DICER 1 somatic or germline variant is unique though the pathologic findings were characteristic of PPBs and mosaicism may provide an explanation for these DICER1 -negative PPBs [9]. Identification of the ETV6-NTRK3 fusion argues this tumor could be molecularly related to an infantile fibrosarcoma but given the brain metastasis, would be an unusual manifestation since the brain is the most common metastatic site for PPB [10]. The tumor continued to progress until his untimely death despite 
the high response rates of NTRK-fused infantile fibrosarcoma to larotrectinib observed in multiple clinical trials [11,12]. TP53 and NRAS pathogenic variants have also been reported to occur frequently in PPB [13] and a TP53 pathogenic variant, p.R273H, was identified in this case. One could hypothesize the TP53 variant modulated response to larotrectinib. Recent evidence from Gatalica et al. showed TP53 is the most commonly co-mutated gene in NTRK-fused neoplasms and another report showed an impressive response to larotrectinib in a refractory high-grade glioma despite tumoral TP53 loss [14,15]. Additionally, the AGO2 p.H443R variant could have promoted resistance given AGO2 is required for the efficient functioning of DICER1. However, this particular mutational change in AGO2 has not been interrogated at the cellular level making its tumoral impact unclear [16]. In this case, earlier identification of the NTRK fusion prior to multiple recurrences and large tumor burden may have improved the chance for a response.

The second case is unique due to the identification of a PIK3CA mutation and MDM2 amplification in addition to a DICER1 germline variant. In the largest published study, exome sequencing was performed on 15 PPBs and none had a PIK3CA mutation or MDM2 amplification [13]. MDM2 is a negative regulator of TP53 $[17,18]$ and its amplification promotes therapeutic resistance leading to poor prognosis in a variety of cancers, including sarcomas, similar to the one in our patient [19-22]. PIK3CA mutations have been reported as potential oncogenic drivers in pediatric rhabdomyosarcoma, a tumor type seen in individuals with pathogenic germline DICER1 variations. [23-26]. Like MDM2, PIK3CA mutations have been shown to promote therapeutic resistance particularly in breast cancer, but also in germ cell tumors and sarcoma [2730]. Early phase trials are currently underway in recurrent pediatric cancers utilizing MDM2 and PIK3CA inhibitors, which stress the need for early identification of these variants to define the utility of these drugs in recurrent $\mathrm{PPB}$.

To date, comprehensive molecular analyses of PPBs are limited. Due to the histologic complexity and heterogeneity of these tumors, these two cases highlight the importance of sequencing all tumors to identify additional oncogenic drivers that promote discovery of early therapeutic interventions for PPB recurrence.

Conflicts of Interest

The authors of this manuscript have no relevant conflicts of interest to disclose.

Acknowledgements

The Precision Genomics Team at Indiana University School of Medicine is supported by U54HD16014 (Renbarger) - Indiana University Center for Pediatric Pharmacology and Precision Medicine (ICPPPM).

The authors would like to acknowledge D. Ashley Hill, MD of Children's National Hospital and the International PPB Registry for reviewing the accuracy of these cases and manuscript.

References

1. Manivel J. C. et al. Pleuropulmonary Blastoma. The so-called pulmonary blastoma of childhood. Cancer. 1988. 62:1516-1526.

2. Messinger Y et al. Pleuropulmonary blastoma: a report on 350 central pathology-confirmed pleuropulmonary blastoma cases by the International Pleuropulmonary Blastoma Registry. 2015. Cancer. 2015. 121(2):276-85.

3. Dehner LP et al. Pleuropulmonary blastoma: evolution of an entity as an entry into a familial tumor predisposition syndrome. Pediatr Dev Pathol. 2015. 18(6):504-511.

4. Nakano Y, et al. Successful treatment of metastatic cerebral recurrence of pleuropulmonary blastoma. Pediatr Blood Cancer. 2019 May;66(5):e27628

5. Hill D.A. et al. DICER1 mutations in familial pleuropulmonary blastoma. Science. 2009. 325:965.

6. Schultz KA et al. DICER1 and associated conditions: Identification of at-risk individuals and recommended surveillance strategies. Clinical Cancer Research 2018. 1-11. 
7. Stewart D.R. et al. Neoplasm risk among individuals with a pathogenic germline variant in DICER1. Journal of Clinical Oncology. 10;37(8)668-676.

8. Priest JR, et al. Cerebral metastasis and other central nervous system complications of pleuropulmonary blastoma. Pediatr Blood Cancer. 2007 Sep;49(3):266-73.

9. Brenneman M, et al. Temporal order of RNase IIIb and loss-of-function mutations during development determines phenotypes in pleuropulmonary blastoma/DICER1 syndrome: a unique variant of the two-hit tumor suppression model. F1000Res. 2015. Jul 10:4:214

10. Parida L, et al. Clinical management of infantile fibrosarcoma: a retrospective single-institution review. Pediatr Surg Int. 2013 Jul;29(7):703-8.

11. Drilon A, et al. Efficacy of Larotrectinib in TRK Fusion-Positive Cancers in Adults and Children. N Engl J Med. 2018 Feb 22;378(8):731-739.

12. Laetsch TW, et al. Larotrectinib for paediatric solid tumours harbouring NTRK gene fusions: phase 1 results from a multicentre, open-label, phase 1/2 study. Lancet Oncol. 2018 May;19(5):705-714.

13. Pugh TJ et al. Exome sequencing of pleuropulmonary blastoma reveals frequent biallelic loss of TP53 and two hits in DICER1 resulting in retention of 5p-derived miRNA hairpin loop sequences. Oncogene. 2014. 33:5295-5302.

14. Gatalica Z, et al. Molecular characterization of cancers with NTRK gene fusions. Mod Pathol. 2019 Jan;32(1):147-153.

15. Ziegler DS, et al. Brief Report: Potent clinical and radiological response to larotrectinib in TRK fusion-driven high-grade glioma. Br J Cancer. 2018 Sep 11; 119(6): 693-696.

16. Koscianska E, et al. The role of Dicer protein partners in the processing of microRNA precursors. PLoS One. 2011;6(12):e28548

17. Momand J, et al. The mdm-2 oncogene product forms a complex with the p53 protein and inhibits p53-mediated transactivation. Cell. 1992 Jun 26;69(7):1237-45.

18. Hou H, et al. The role of MDM2 amplification and overexpression in therapeutic resistance of malignant tumors. Cancer Cell Int. 2019 Aug 22;19:216.

19. Kato S, et al. Analysis of MDM2 Amplification: Next-Generation Sequencing of Patients With Diverse Malignancies. JCO Precis Oncol. 2018;2018.

20. Dembla V, et al. Prevalence of MDM2 amplification and coalterations in 523 advanced cancer patients in the MD Anderson phase 1 clinic. Oncotarget. 2018 Sep 4;9(69):33232-33243.

21. Sawada R, et al. MDM2 copy number increase: a poor prognostic, molecular event in esophageal squamous cell carcinoma. Hum Pathol. 2019 Jul;89:1-9.

22. Bill KLJ, et al. Degree of MDM2 Amplification Affects Clinical Outcomes in Dedifferentiated Liposarcoma. Oncologist. 2019 Jul;24(7):989-996.

23. Shukla N, et al. Oncogene mutation profiling of pediatric solid tumors reveals significant subsets of embryonal rhabdomyosarcoma and neuroblastoma with mutated genes in growth signaling pathways. Clin Cancer Res. 2012 Feb 1;18(3):748-57.

24. Kikuchi K, et al. MDM2 Amplification and PI3KCA Mutation in a Case of Sclerosing Rhabdomyosarcoma. Sarcoma. 2013;2013:520858.

25. Agaram NP, et al. MYOD1-mutant spindle cell and sclerosing rhabdomyosarcoma: an aggressive subtype irrespective of age. A reappraisal for molecular classification and risk stratification. Mod Pathol. 2019 Jan;32(1):27-36. 
26. Doros L, et al. DICER1 mutations in embryonal rhabdomyosarcomas from children with and without familial PPB-tumor predisposition syndrome. Pediatr Blood Cancer. 2012 Sep;59(3):558-60.

27. Lobil S, et al. PIK3CA mutations are associated with reduced pathological complete response rates in primary HER2-positive breast cancer: pooled analysis of 967 patients from five prospective trials investigating lapatinib and trastuzumab. Ann Oncol. 2016 Aug;27(8):1519-25.

28. Rimawi MF, et al. Low PTEN levels and PIK3CA mutations predict resistance to neoadjuvant lapatinib and trastuzumab without chemotherapy in patients with HER2 over-expressing breast cancer. Breast Cancer Res Treat. 2018 Feb;167(3):731-740.

29. Feldman DR, et al. Presence of somatic mutations within PIK3CA, AKT, RAS, and FGFR3 but not BRAF in cisplatin-resistant germ cell tumors. Clin Cancer Res. 2014 Jul 15;20(14):3712-20.

30. Jiang Y, et al. Novel secondary somatic mutations in Ewing's sarcoma and desmoplastic small round cell tumors. PLoS One. 2014 Aug 13;9(8):e93676.

Table 1 Legend

*No pathogenic DICER1 variants were found either tumor sample despite reanalysis.

**The ETV6-NRTK3 gene fusion in the initial sequencing was later identified upon reanalysis of the sequencing data.

*** Germline analysis was performed at Ambry Genetics Laboratory as FoundationOne testing does not offer germline analysis

Definitions: VUS = Variant of Unknown Significance. A variant of unknown significance is an allele, or variant form of a gene, whose significance to the normal function of the encoded protein and any corresponding phenotype, is unknown. WT $=$ Wild Type

\section{Hosted file}

Table 1 Final.docx available at https://authorea.com/users/323782/articles/452293previously-unreported-somatic-variants-in-two-patients-with-pleuropulmonary-blastomawith-metastatic-brain-recurrence 